\title{
MINIMAL CYCLOTOMIC SPLITTING FIELDS FOR GROUP CHARACTERS
}

\author{
R. A. MOLLIN ${ }^{1}$
}

\begin{abstract}
Let $F$ be a finite Galois extension of the rational number field $Q$, and let $G$ be a finite group of exponent $n$ with absolutely irreducible character $\chi$. This paper provides sufficient conditions for the existence of a minimal degree splitting field $L$ with $F(\chi) \subseteq L \subseteq F\left(\varepsilon_{n}\right)$, where $\varepsilon_{n}$ is a primitive $n$th root of unity. We obtain as immediate corollaries known results pertaining to this question in the literature. Moreover we obtain necessary and sufficient conditions for the existence of a minimal splitting field $L$ as above which is cyclic over $F(\chi)$. The machinery we use to achieve the above results are certain genus numbers of $F(\chi)$.
\end{abstract}

1. Introduction. Let $F$ be a finite Galois extension of $Q, G$ a finite group of exponent $n, \chi$ a complex irreducible character of $G$, and let $A(\chi, F)$ denote the simple component of $F G$ corresponding to $\chi$. A finite extension $L$ of $F(\chi)$ is a splitting field for $\chi$ over $F$ if the class of $A(\chi, F) \otimes_{F(\chi)} L$ is equivalent to $L$ in the Brauer group $B(L)$ of $L$. The minimum of the degrees $|L: F(\chi)|$ of $L$ over $F(\chi)$ taken over all splitting fields $L$ of $\chi$ is the Schur index $m_{F}(\chi)$ of $\chi$ over $F$. It is the purpose of this paper to provide sufficient conditions for the existence of a splitting field $L$ of $\chi$ such that $F(\chi) \subseteq L \subseteq F\left(\varepsilon_{n}\right)$ and $|L: F(\chi)|=m_{F}(\chi)$. Under a suitable restriction we generalize this to a field $F$ of characteristic zero. Moreover, we are able to provide necessary and sufficient conditions for such an $L$ to exist, where $L$ is cyclic over $F(\chi)$.

The above results continue work begun in [Mo 1] and advances $[$ Fe 1], [Fe 2] and the more recent $[\mathbf{S p}-\mathbf{T}]$.

2. Notation and preliminaries. Relevant notation or concepts not discussed may be found in [Mo 1]. Let $K$ be a finite Galois extension of an algebraic number field $F$ with Galois group $G(K / F)$. When $G(K / F)$ is abelian we adopt the convention of [Mo 1] with respect to decomposition of primes; i.e. if $\hat{p}$ is a $K$-prime above the $F$-prime $p$ then any reference to the decomposition of $\hat{p}$ in $K$ over $F$ shall be made instead to the decomposition of $p$ in $K / F$. Moreover, in this case we write $K_{p}$ for $K_{\hat{p}}$, the completion of $K$ at $\hat{p}$.

Now let $A(\chi, F)$ be as in $\S 1$; then if $\hat{q}$ and $q^{\prime}$ are $F(\chi)$-primes above the same rational prime $q$ then $A(\chi, F) \otimes_{F(\chi)} F(\chi)_{\hat{q}}$ and $A(\chi, F) \otimes_{F(\chi)} F(\chi)_{q^{\prime}}$ have the same index (we proved this in [Mo 2] -[Mo 3] as a generalization of [Be]). Denote the common value of all indices of $A(\chi, F) \otimes_{F(\chi)} F(\chi)_{\hat{q}}$ for all $F(\chi)$-primes $\hat{q}$ above a given rational prime $q$ by ind $(A(\chi, F))$ called the $q$ local index of $A(\chi, F)$.

Received by the editors April 25, 1983.

1980 Mathematics Subject Classification. Primary 20 C05.

${ }^{1}$ The author's research is supported by N.S.E.R.C. Canada.

(c) 1984 American Mathematical Society $0002-9939 / 84 \$ 1.00+\$ .25$ per page 
For an algebraic number field $F$ we denote the genus field of $F$ by $\tilde{F}$ which is defined as the maximal abelian extension of $F$, such that $\tilde{F}$ is the composite of an abelian extension of $Q$ with $F$ and is unramified at all finite primes (see [Ish] for details). $|\tilde{F}: F|=g(F)$ is called the genus number of $F$. We define the $n$th genus field for a given positive integer $n$ as $\tilde{F}^{(n)}=\tilde{F} \cap F\left(\varepsilon_{n}\right)$. We call $\left|\tilde{F}^{(n)}: F\right|=g_{n}(F)$ the $n$th genus number of $F$. It is this number which will provide the machinery for the major result of this paper.

\section{Splitting fields.}

LEMMA 1. Let $F$ be a finite Galois extension of $Q$, and let $n$ be a positive integer. Suppose that $G=G\left(F\left(\varepsilon_{n}\right) / F\right)$ is cyclic of prime power order. Then we have that $g_{n}(F)=\left|T^{(q)}: F\right|$ where $T^{(q)}$ is the inertia field of an $F$-prime $q$ having nontrivial ramification in $F\left(\varepsilon_{n}\right)$. Furthermore if $n$ is divisible by at least two distinct primes then $g_{n}(F)=\left|Z^{(q)}: F\right|$ where $Z^{(q)}$ is the decomposition subfield of $F\left(\varepsilon_{n}\right)$ over $F$ at $q$.

Proof. The intertia subgroup $I^{(q)}$ of $G$ at $q$ is contained in $G\left(F\left(\varepsilon_{n}\right) / F(\varepsilon)\right)$ where $\varepsilon$ is a root of unity in $F\left(\varepsilon_{n}\right)$ having largest possible order relatively prime to $p=q \cap Q$ (see [Nark, Theorem 5.9, p. 210]). Since $G$ is cyclic of prime power order it follows that only $F$-primes above $p$ may ramify in $F\left(\varepsilon_{n}\right)$. Since $F$ is Galois over $Q$ then $I^{(q)}=I^{\left(q^{\prime}\right)}$ for any $F$-primes $q$ and $q^{\prime}$ above $p$. Hence $g_{n}(F)=\left|T^{(q)}: F\right|$.

Now suppose $n=q^{a} t$ where $q$ and $t$ are relatively prime, $t>1$, and $q$ lies above $p$. Then $F_{q}\left(\varepsilon_{t}\right)$ is nontrivial cyclic unramified over $F_{q}$ if $Z^{(q)} \neq T^{(q)}$. Moreover by hypothesis $G\left(F_{q}\left(\varepsilon_{q^{a}}\right) / F_{q}\right)$ is nontrivial. Hence $G\left(F_{q}\left(\varepsilon_{n}\right) / F_{q}\right)$ is generated by at least 2 elements. Since $G\left(F_{q}\left(\varepsilon_{n}\right) / F_{q}\right) \cong G\left(F\left(\varepsilon_{n}\right) / Z^{(q)}\right)$, the decomposition subgroup of $G$ at $q$, then $G$ is not cyclic, a contradiction. Hence $g_{n}(F)=$ $\left|Z^{(q)}: F\right|$.

Now we set the stage for the main result. Let $\chi$ be a complex irreducible character of a finite group of exponent $n$, and set $A=A(\chi, F)$ where $F$ is finite Galois over $Q$. Let $S(A)$ be defined as the set of all rational primes $q$ such that $\operatorname{ind}_{q}(A)>1$. We now define, for convenience sake, a field $L$ to have $(n, F)$-splitting property provided that $L$ is a splitting field of $\chi$ such that $F(\chi) \subseteq L \subseteq F\left(\varepsilon_{n}\right)$ and $|L: F(\chi)|=m_{F}(\chi)$. Moroever let $K=F(\chi)$ henceforth.

THEOREM 1. Let $\chi$ be a complex irreducible character of a finite group of exponent $n$, and set $A=A(\chi, F)$ where $F$ is a finite Galois extension of $Q$ such that if $K$ is real then $2 \notin S(A)$. If for all finite odd $q \in S(A)$ we have that $\left|g_{q}(K)\right|_{p} \leq\left|m_{F}(\chi) / \operatorname{ind}_{q}(A)\right|_{p}$ for each $p \mid m_{F}(\chi)$ then there exists an $L$ with $(n, F)$ splitting property.

PROOF. By the same reasoning as in the proof of [Mo 1] we may assume that $m_{F}(\chi)=p^{a}$ where $p$ is prime.

Let $q \in S(A)$ where $q$ is finite odd. Suppose furthermore that $\operatorname{ind}_{q}(A)=p^{b}$. From [Ya, Theorem 4.7, p. 46] we may deduce that there exists a subfield $M^{(q)} \subseteq$ $K\left(\varepsilon_{q}\right)$ such that $\left|M_{\hat{q}}^{(q)}: K_{\hat{q}}\right|=\operatorname{ind}_{q}(A)$ where $\hat{q}$ is any $K$-prime above $q$. However

$$
\left|M^{(q)}: K\right|=\left|M^{(q)}: T^{(\hat{q})}\right|\left|T^{(\hat{q})}: K\right|=p^{b}\left|T^{(\hat{q})}: K\right|=p^{b}\left|g_{q}(K)\right|_{p}
$$

by Lemma 1 , where $T^{(\hat{q})}$ is the inertia subfield of $K\left(\varepsilon_{q}\right)$ over $K$ at $\hat{q}$. Thus by hypothesis we have $\left|M^{(q)}: K\right| \leq p^{a}$. 
Therefore, for each finite odd $q \in S(A)$ we have a field $M^{(q)}$ such that $M^{(q)}$ splits $\chi$ at $q$ and $\left|M^{(q)}: K\right| \leq p^{a}$. By [Ya, Theorem 6.2, p. 89] we have that $\varepsilon_{p^{a}}$ is in $K$; so $M^{(q)}=K(\theta(q))$ where $\theta(q)^{p^{a}} \in K$.

Now we construct $L$ according to the contents of $S(A)$.

Case (1). If all $q \in S(A)$ are finite odd then choose $\alpha$ as in [Mo 5]. Therefore $\left|K_{\hat{q}}(\alpha): K_{\hat{q}}\right|=\operatorname{ind}_{q}(A)$ for all $q \in S(A)$ where $\hat{q}$ is any $K$-prime above $q$. However there exists a $q \in S(A)$ with $\operatorname{ind}_{q}(A)=p^{a}$ so $L=K(\alpha)$ is the required field.

Case 2. If $2 \in S(A)$ then by hypothesis $K$ is nonreal and thus only finite primes are contained in $S(A)$. By [Ya], $p^{a}=2$ and $\sqrt{-1}$ is not in $K$. Now we choose $\alpha$ as in the proof of [Mo 1, Theorem 1, p. 108]. Then $L=K(\alpha)$ is the required field.

Case 3. If $q \in S(A)$ is inifinite then $K$ must be real so that by hypothesis $2 \notin S(A)$. Let $\alpha^{\prime}=\prod \theta(q)$ where the product ranges over all finite primes $q$ in $S(A)$. If $K\left(\alpha^{\prime}\right)$ is nonreal then choose $\alpha=\alpha^{\prime}$ and choose $\alpha=\sqrt{-1} \cdot \alpha^{\prime}$ otherwise. Hence $L=K(\alpha)$ is the required field.

Now we give a sequence of applications of Theorem 1. We anchor them to the theorem as corollaries thereof and for each corollary we maintain the first statement of Theorem 1 as being in force.

Under a suitable restriction we may generalize Theorem 1 to a field $F$ of characteristic zero.

COROLLARY 1. Let $F$ be a field of characteristic zero such that $m_{F}(\chi)=$ $m_{F^{\prime}}(\chi)$ where $F^{\prime}=F \cap Q\left(\varepsilon_{n}\right)$. Then there exists a field with $(n, F)$ splitting property if and only if there exists a field with $\left(n, F^{\prime}\right)$ splitting property.

Proof. By [Mo 4, Theorem 3.4, p. 473] we have $A(\chi, F) \cong A(\chi, Q) \otimes_{Q(\chi)} K$. When $m_{F}(\chi)=m_{F^{\prime}}(\chi)$ the result clearly follows. (Note that in general we always have $m_{F}(\chi) \mid m_{F^{\prime}}(\chi)$.)

The following generalizes [Sp-T, Corollary 5, p. 36] (see also [Mo 1, Corollary 1, p. 110]).

COROLLARY 2. If $g(K)$ and $m_{F}(\chi)$ are relatively prime then there is a field with $(n, F)$-splitting property.

Proof. For each prime $p$ dividing $m_{F}(\chi)$ we have $|g(K)|_{p}=1$. Hence $\left|g_{q}(K)\right|_{p}$ $=1$ for each $q \in S(A)$.

We note that the converse of the above fails. We provide the following counterexample (which corrects [Mo 1, Example 2, p. 111] which was missed in [Mo 5]).

EXAMPLE 1. Let $p$ be an aribtrary odd prime and $q$ a prime with $q \equiv 1$ $\left(\bmod p^{4}\right)$ but $q \not \equiv 1\left(\bmod p^{5}\right)$. Let

$$
\langle\sigma\rangle=G\left(Q\left(\varepsilon_{p^{3} q}\right) / Q\left(\varepsilon_{p^{3}}\right)\right) \quad \text { and }\langle\tau\rangle=G\left(Q\left(\varepsilon_{p^{3} q}\right) / Q\left(\varepsilon_{q}\right)\right) .
$$

Set $\gamma=\sigma^{\left((q-1) / p^{4}\right)} \tau^{p(p-1)}$ and let $K$ be the fixed field of $\langle\gamma\rangle$.

Let $G=\left\langle x, y, z: x^{q}=z^{p^{3}}=1, y^{p^{4}}=z^{p}, z^{p}\right.$ central, $y z y^{-1}=z^{a}$ and $y x y^{-1}=$ $\left.x^{b}\right\rangle$ where $\gamma: \varepsilon_{p^{3}} \rightarrow \varepsilon_{p^{3}}^{a}$ and $\gamma: \varepsilon_{q} \rightarrow \varepsilon_{q}^{b}$. Set $A=\left(Q\left(\varepsilon_{p^{3} q}\right) / K, \varepsilon_{p^{2}}\right)$, a crossed product algebra (see [Mo 1]) which is a homomorphic image of $Q G$. Therefore there is a complex irreducible character $\chi$ of $G$ with $A=A(\chi, Q)$, and $K=Q(\chi)$. As in $\left[\right.$ Mo 1], $\operatorname{ind}_{q}(A)=p=m_{Q}(\chi)$ and in fact $S(A)=\{q\}$. 
Also since $|G|=p^{6} q=n$ then by $[$ Fe 1, Theorem 6, p. 429] there is a field with $(n, Q)$-splitting property. However $\tilde{K}=Q\left(\varepsilon_{p^{3}}, \theta\right)$ where $Q(\theta)$ is the unique subfield of $Q\left(\varepsilon_{q}\right)$ of degree $(q-1) / p^{3}$ over $Q$; i.e. $p \mid g(K)$. This completes the example.

The following generalizes [Sp-T, Corollary 7, p. 36].

COROLlaRY 3. Let $F\left(\varepsilon_{r}\right)$ be the smallest root of unity field with $K \subseteq F\left(\varepsilon_{r}\right)$. Suppose $p$ does not divide the ramification index of any $\hat{q}$ above $q \in S(A)$ in $F\left(\varepsilon_{r}\right)$ over $K$, for each $p \mid m_{F}(X)$. Then there is a field with $(n, F)$-splitting property.

Proof. As in the proof of [Sp-T, Corollary 7, p. 36] we get $\left|K_{\hat{q}}\left(\varepsilon_{q}\right): K_{\hat{q}}\right|=$ $\left|K\left(\varepsilon_{q}\right): K\right|$. Hence $g_{q}(K)=1$.

The following corollary which is immediate from the theorem generalizes the main result of [Mo 1, Theorem 1, p. 108].

COROLLARY 4. If $K$ is totally nonreal over $Q$ and if for each prime $p \mid m_{F}(\chi)$ we have $\left|K\left(\varepsilon_{q}\right): K\right|_{p}=\left|K_{\hat{q}}\left(\varepsilon_{q}\right): K_{\hat{q}}\right|_{p}$ whenever $\hat{q}$ is $K$-prime above an odd prime $q \in S(A)$ then there is a field with $(n, F)$-splitting property.

The following uses Corollaries 3 and 4 to give a result which generalizes [Sp-T, Corollary 6, p. 36] which in turn generalized [Mo 1, Corollary 1, p. 110]. $\varepsilon_{r}$ is as defined in Corollary 3.

COROLlaRY 5. Suppose that $1=\left(m_{F}(\chi),\left|F\left(\varepsilon_{r}\right): K\right|, g(K)\right)$. Then there is a field with $(n, F)$-splitting property.

PROOF. If

$$
\left(m_{F}(\chi), g(K)\right)=1
$$

then we proceed as in Corollary 2. If $p \mid\left(m_{F}(\chi), g(K)\right)$ then $p \nmid\left|F\left(\varepsilon_{r}\right): K\right|$ and we proceed as in Corollary 3.

The next corollary generalizes the main result of [Sp-T, Theorem 3, p. 35].

COROLlaRY 6. Suppose that whenever $q \in S(A)$ and $p \mid \operatorname{ind}_{q}(A)$ we have $\left|T^{(\hat{q})}: K\right|_{p}=1$ where $T^{(\hat{q})}$ is the inertia subfield of $K\left(\varepsilon_{q}\right)$ over $K$ at $\hat{q}$ which lies over $q$. Then there is a field with $(n, F)$-splitting property.

PROOF. The hypothesis forces $\left|g_{q}(K)\right|_{p}=1$.

The converse of Corollary 6 fails as shown in

EXAMPLE 2. Take $p=3$ and $q=163$ in Example 1. Then $K\left(\varepsilon_{q}\right)=Q\left(\varepsilon_{3^{3} \cdot 163}\right)$ and $\left|K\left(\varepsilon_{q}\right): K\right|=3^{4}$ which is greater than $\left|K_{q}\left(\varepsilon_{q}\right): K_{q}\right|=3^{3}$ since 163 splits in $\tilde{K}=Q\left(\theta, \varepsilon_{p^{3}}\right)$ as defined in Example 1. Now as in Example 1 we get $K=Q(\chi)$ for a complex irreducible character $\chi$ of $G$ and an $A=A(\chi, Q)$ with $\operatorname{ind}_{q}(A)=$ $p=m_{Q}(\chi)$. Moreover there is a field with $(n, F)$-splitting property where $n$ is the exponent of $G$. This secures the example.

Now we are able to achieve necessary and sufficient conditions for the existence of a field with $(n, F)$-splitting property which is cyclic over $F(\chi)$.

THEOREM 2. Let $\chi, A, S(A), G, n, F$ and $K$ be as above. Then there is a field $L$ with $(n, F)$-splitting property such that $L$ is cyclic over $K$ if and only if for each $p$ dividing $m_{F}(\chi)$ there is a decomposition $G_{p}=G\left(K\left(\varepsilon_{n}\right) / K\right)_{p}$ as a direct product of cyclic groups $C_{i}$ with fixed field $K_{i}$ such that for some $i$, say $i=1$, we have that 
for all $q \in S(A),\left|Z_{1}^{(q)}: K_{1}\right| \leq\left|m_{F}(\chi) / \operatorname{ind}_{q}(A)\right|_{p}$ and $\left|K\left(\varepsilon_{n}\right): K_{1}\right| \geq\left|m_{F}(\chi)\right|_{p}$ where $Z_{1}^{(q)}$ is the decomposition subfield of $K\left(\varepsilon_{n}\right)$ over $K_{1}$ at $q$.

Proof. If such an $L$ exists then, since $L$ is cyclic over $K$, there is a decomposition such that $L \subseteq \bigcap_{i>1} K_{i}$ after possibly renumbering the $K_{i}$. Now since $Z_{1}^{(q)} \cap L$ is the $p$-part of the decomposition subfield of $L$ over $K$ at $q$ for each $q \in S(A)$ then it follows that $\left|Z_{1}^{(q)}: K_{1}\right| \leq\left|m_{F}(\chi) / \operatorname{ind}_{q}(A)\right|_{p}$. Moreover since $\left|K\left(\varepsilon_{n}\right): K_{1}\right| \geq|L: K|_{p}$ then $\left|K\left(\varepsilon_{n}\right): K_{1}\right| \geq\left|m_{F}(\chi)\right|_{p}$.

Conversely, suppose that we have such a decomposition. Then we may choose $L \subseteq \bigcap_{i>1} K_{i}$ such that $\left|L_{q}: K_{q}\right|=\left|\operatorname{ind}_{q}(A)\right|_{p}$ for each $q \in S(A)$. Now for some $q \in$ $S(A)$ we have that $\left|\operatorname{ind}_{q}(A)\right|_{p}=\left|m_{F}(\chi)\right|_{p}$ and for this $q$ we have $Z_{1}^{(q)}=K_{1}$. Since $Z_{1}^{(q)} \cap L=K$ is the decomposition subfield of $L$ at $q$ then $|L: K|=\left|m_{F}(\chi)\right|_{p}$.

Now as a consequence of Theorem 2 we easily generalize [Fe $\mathbf{1}$, Theorem (b), p. 429] which we could not accomplish in [Mo 1]. The above notation remains in force.

COROLlaRY 7. Suppose $m_{F}(\chi) \geq 3$ and $n=p^{a} q^{b}$ for primes $p<q$ then there exists a cyclic field with $(n, F)$-splitting property.

Proof. By [Gold-Is] (see also [Mo 6]) $G_{p}=G\left(K\left(\varepsilon_{n}\right) / K\right)_{p}$ is not cyclic. Now since $K\left(\varepsilon_{p^{a}}\right)$ is cyclic over $K$ then there is a decomposition of $G_{p}$ as a product of cyclic groups $C_{1} \times C_{2}$ with $K\left(\varepsilon_{n}\right)$ totally ramified over $K_{1}$ at $q$.

\section{REFERENCES}

[Be] M. Benard, The Schur subgroup. I, J. Algebra 22 (1972), 374-377.

[Cor] G. Cornell, Abhyankar's lemma and the class group (Proc. Illinois Number Theory Conf.), Lecture Notes in Math., Vol. 751, Springer-Verlag, Berlin and New York, 1979, pp. 82-88.

[Fe 1] B. Fein, Minimal splitting fields for group representations, Pacific J. Math. 51 (1974), 427-431.

[Fe 2] _ Minimal splitting fields for group representations. II, Pacific J. Math. 77 (1978), 445-449.

[Gold-Is] D. Goldschmidt and I. Isaacs, Schur indices in finite groups, J. Algebra 33 (2) (1975), 191-199.

[Ish] M. Ishida, The genus fields of algebraic number fields, Lecture Notes in Math., Vol. 555, SpringerVerlag, Berlin and New York, 1976.

[Mo 1] R. Mollin, Splitting fields and group characters, J. Reine Angew. Math. 315 (1980), 107-114.

[Mo 2] _ Generalized uniform distribution of Hasse invariants, Comm. Algebra 5 (1977), 245-266.

[Mo 3] _ Uniformly distrbuted Hasse invariant, preprint.

[Mo 4] _ , The Schur group of a field of characteristic zero, Pacific J. Math. 76 (1978), 471-478.

[Mo 5] _ , Correction to the paper: Splitting fields and group characters, J. Reine Angew. Math. 327 (1981), 219-220.

[Mo 6] Schur indices, sums of squares and splitting fields, C. R. Math. Rep. Acad. Sci. Canada 3 (1981), 301-306.

[Nark] W. Narkiewicz, Elementary and analytic theory of algebraic numbers, Polish Scientific Publishers, Warsaw, 1974.

[Rib] P. Ribenboim, Algebraic numbers, Wiley Interscience, New York, 1972.

[Sp-T] E. Spiegel and A. Trojan, Minimal splitting fields in cyclotomic extensions, Proc. Amer. Math. Soc. 87 (1983), 33-37.

[Ya] T. Yamada, The Schur subgroup of the Brauer group, Lecture Notes in Math., Vol. 397, SpringerVerlag, Berlin and New York, 1974. 